\title{
Electron Microscopy of Morphed Graphene Nanostructures Synthesized by Mechanical Milling
}

\author{
H. A. Calderon ${ }^{1}$, F. Alvarez Ramírez², I. Estrada Guel ${ }^{3,4}$, V. G. Hadjiev³ , F.C. Robles Hernandez ${ }^{3}$. \\ 1. Departamento de Física, ESFM-IPN, Zacatenco, Ciudad de Mexico, Mexico. \\ 2. Instituto Mexicano del Petróleo, Ciudad de Mexico, México \\ 3. Department of Mech. Eng. Technology, University of Houston, Houston, TX, USA. \\ 4. Centro de Investigación en Materiales Avanzados, Chihuahua, México.
}

There are many carbon nanostructures (CN's) and most likely graphene and diamond are still the most interesting due to its physical properties. Synthesis of CN's is made via diverse methods including vapor deposition, exfoliation (graphene) or the use of high pressure and temperature (diamond). Thus it is interesting to investigate a purely mechanical, simple and room temperature procedure to synthesize CN's. This investigation deals with the products of mechanically milling carbon soot at room temperature that has rendered a different $\mathrm{C}$ allotrope herein identified as morphed graphene. Identification of a new allotrope is quite complicated due to the wide variety of known carbon phases and the pronounce effects of even subtle morphology changes as in the twisted layer graphene. Nevertheless different forms of evidence have been shown that lead to conclude that the product of the synthesis procedure in use (mechanical milling) indeed delivers a new allotrope [1]. Starting with carbon soot, milling develops a variety of phases ranging from graphene (single, double-layered) to diamond. Similar phases have been previously proposed from theoretical considerations and numerical calculations [2]. In this work, medium dose electron microscopy is used to an in-depth characterization of the product phases and especially this newly found allotrope known as Rh6 and Rh6-II having a pure sp2 bonding.

Figure 1 shows two experimental images and a phase image. This is the result of an exit wave

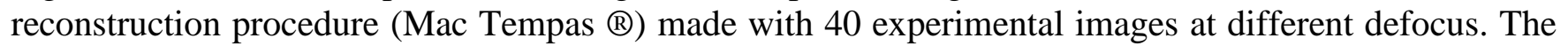
procedure allows using relatively lower dose rates while keeping atomic resolution. Phases of interest are made evident by using Fourier transforms (FFT equivalent in this case to diffraction patterns) and selecting specific reflections according to the lattice spacings that the FFT contains. The complete FFT is given in Figure 2 together with all lattice spacings involved. Figure 3 shows a section of the phase image and the corresponding Fourier analysis that results in the identification of phase Rh6 i.e., a new allotrope of C. Figure 4 shows the corresponding analysis that leads to identify the allotrope Rh6 II. These allotropes have never before been synthesized but mechanical milling with its high entropy has made it possible. [3]

\section{References:}

[1] H.A. Calderon et al, Accepted for publication by Carbon.

[2] J. - T Wang et al, Scientific Reports 4 (2014) p. 4339.

[3] The authors acknowledge funding from the IPN, CONACYT and COFFA IPN. The use of TEAM 05 at NCEM-LBNL is supported by the Molecular Foundry, which is supported by the Office of Science, the Office of Basic Energy Sciences, the U.S. Department of Energy under Contract No. DE-AC02$05 \mathrm{CH} 11231$. 


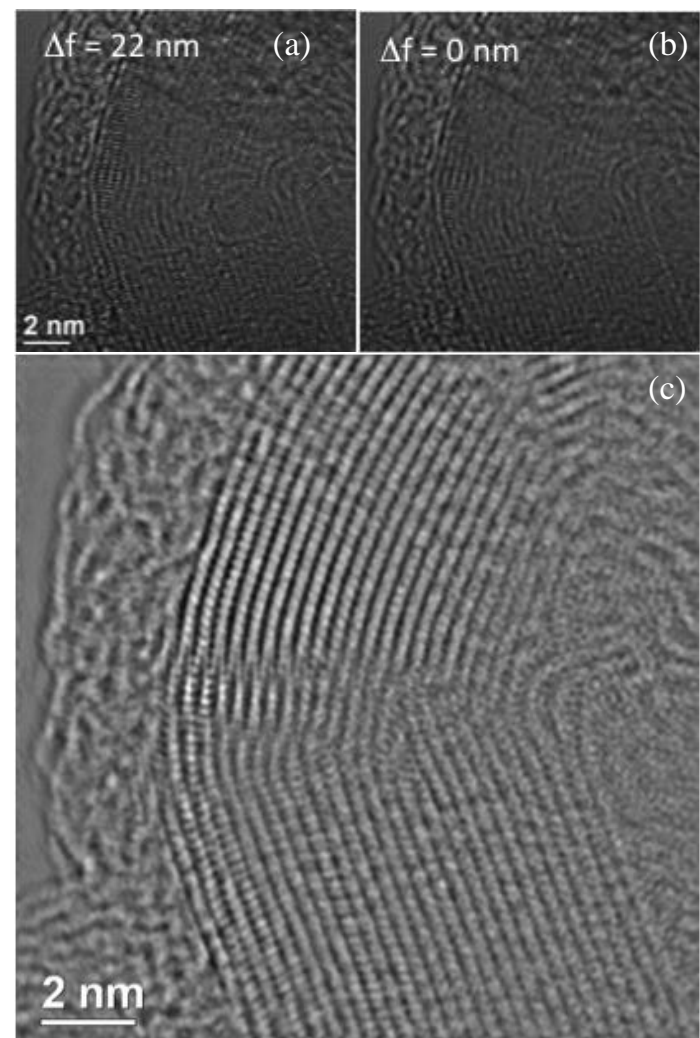

Figure 1. Experimental (a,b) and phase images (c) of $\mathrm{C}$ phases after mechanical milling.

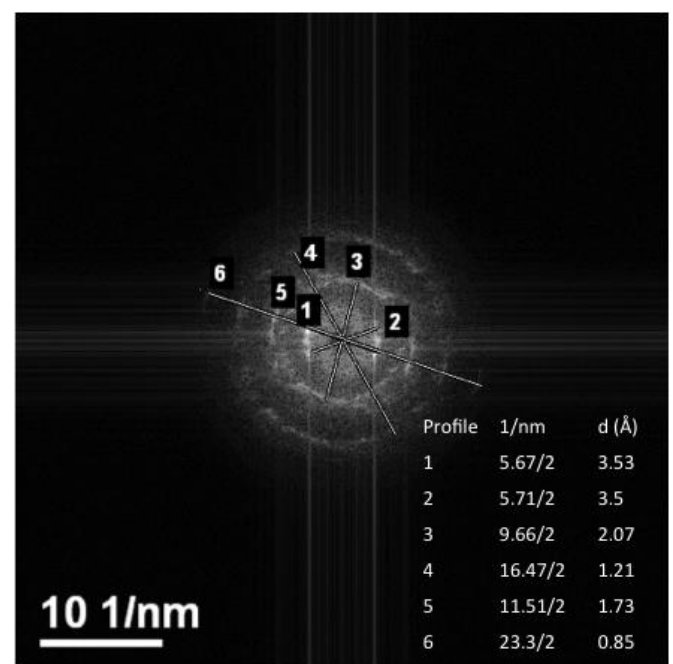

Figure 2. FFT of phase image shown in Fig. 1.
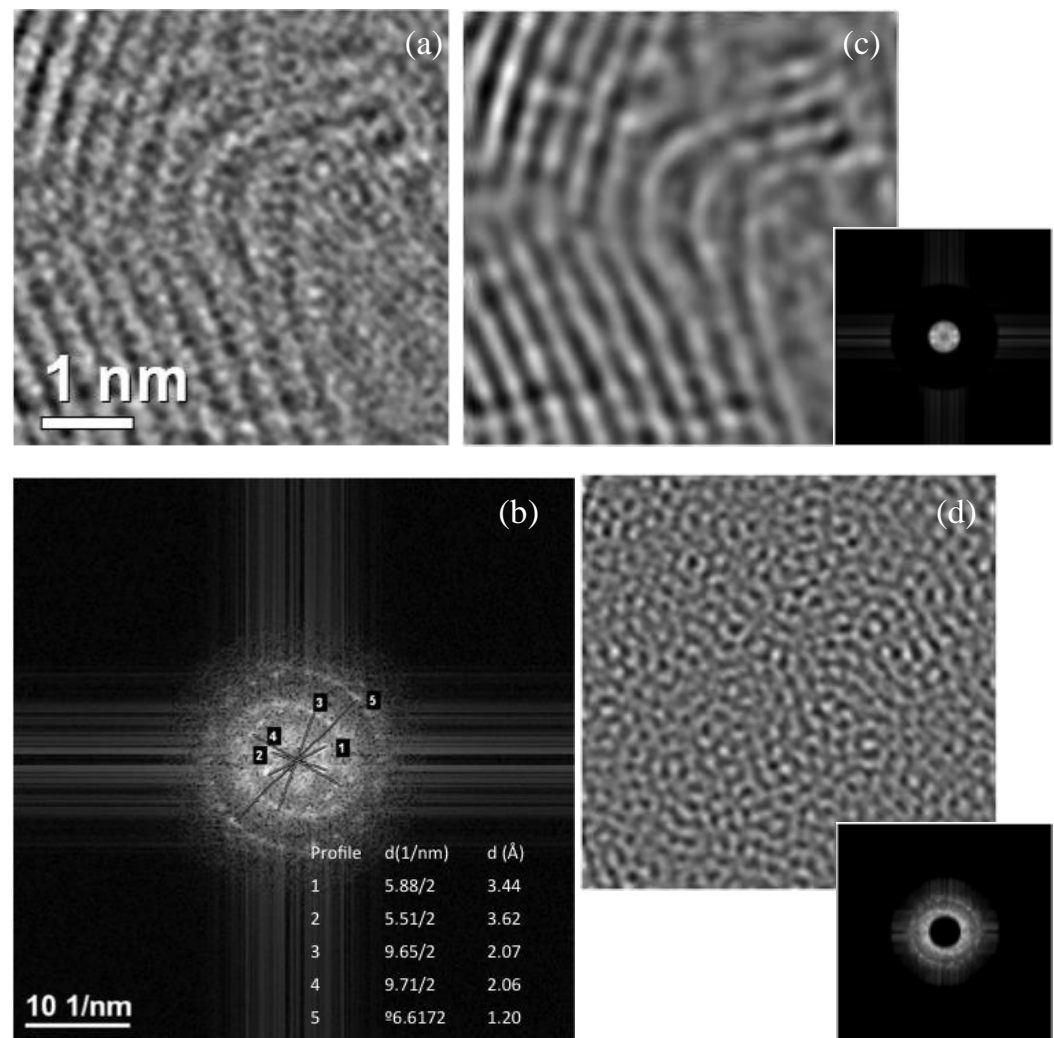

Figure 3. Identification of Phase Rh6. (a) Section of phase image, (b) corresponding FFT with measured lattice spacings and $(\mathrm{c}, \mathrm{d})$ selected IFFTs.
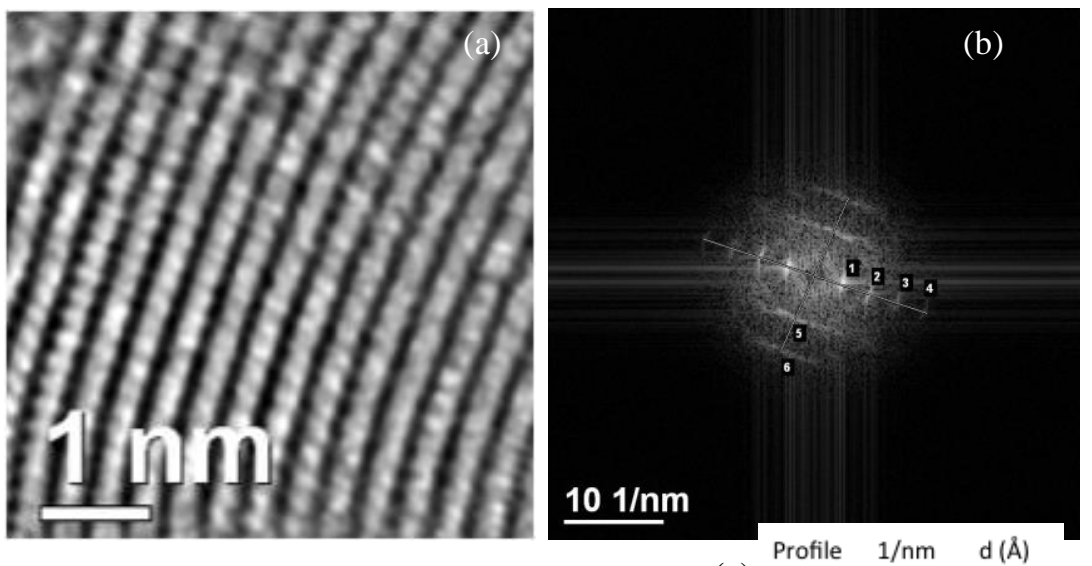

Figure 4. Identification of Phase Rh6 II. (a) Section of Phase image, (b) corresponding FFT with measured lattice spacings as given in Table (c). (c)

$\begin{array}{lll}1 & 5.61 / 2 & 3.56 \\ 2 & 11.6 / 2 & 1.72 \\ 3 & 17.3 / 2 & 1.16 \\ 4 & 23.1 / 2 & 0.86 \\ 5 & 9.6 / 2 & 2.08 \\ 6 & 16.3 / 2 & 1.23\end{array}$

